\title{
Growth of Tyzzer's Organism in Primary Monolayer Cultures of Adult Mouse Hepatocytes
}

\author{
By S. KAWAMURA, F. TAGUCHI, T. ISHIDA, M. NAKAYAMA AND \\ K. FUJIWARA* \\ Department of Animal Pathology, Institute of Medical Science, University of Tokyo, \\ Shirokanedai, Minato-ku, Tokyo 108, Japan
}

(Received 14 May 1982; revised 30 July 1982)

\begin{abstract}
The Tyzzer's disease organism was grown in primary monolayer cultures of adult mouse hepatocytes prepared by collagenase perfusion. The organisms produced a plaque-like cytopathic effect involving almost the whole culture around $72 \mathrm{~h}$ post-infection when the bacterial growth reached a maximum. The organisms showed specific immunofluorescence, and electron microscopy revealed that intracellular organisms had peritrichous flagella and underwent cell division. After intravenous inoculation of the infected cell culture into mice, necrotic hepatitis was produced and the organisms, recovered from the liver lesion, could be propagated in primary culture of mouse hepatocytes.
\end{abstract}

\section{INTRODUCTION}

Tyzzer's disease of laboratory mice is characterized by multiple focal necrosis in the liver and the presence of slender bacilli within hepatocytes (Tyzzer, 1917). The same disease has also been noticed in other species of laboratory, domestic and wild animals (Fujiwara, 1978). Because culture of the causative agent in vitro is still unattainable, information on its basic microbiology is lacking and its taxonomic position has remained undetermined. Successful propagation of the organism was described either in embryonic mouse tissue placed on agar slopes by Rights et al. (1947), or on agar medium containing mouse liver homogenate by Kanazawa \& Imai (1959), but neither report was confirmed. Other than mouse passage, growth in chick embryos has been the only system available for propagating the organism (Fujiwara et al., 1963; Craigie, 1966; Ganaway et al., 1971 $a, b$ ). In order to elucidate the biological nature of the causative agent as well as its pathogenic mechanisms, in vitro culture of the organism would be of great value.

Recently, primary monolayer cultures of adult rat hepatocytes prepared by enzymic perfusion have been used to study hepatocyte physiology. The method was also adapted to the mouse to study hepatotropic virus infections (Arnheiter, 1980). The present report describes the successful growth of the Tyzzer's organism in a primary monolayer culture of adult mouse hepatocytes prepared by the same procedure.

\section{METHODS}

Animals. Female ICR mice (5 weeks old) obtained from a commercial breeder were used to prepare infective material and immune sera, and for pathogenicity tests. ICR mice (8-13 weeks old) from the same source were used to prepare hepatocyte cultures. The breeder colony had been proved by seromonitoring to be free from the main murine pathogens: Tyzzer's organism, Corynebacterium kutscheri, Bordetella bronchiseptica, Mycoplasma pulmonis, Salmonella typhimurium, murine hepatitis virus and Sendai virus (Fujiwara, 1971).

Hepatocyte cultures. Hepatocyte cultures were prepared by a modification of the methods described by Seglen (1976) and Arnheiter (1980). Mice were anaesthetized with Nembutal [50 $\mathrm{mg}$ (kg body weight) ${ }^{-1}$ ] and the liver, in situ, was perfused via the portal vein with modified Hanks' solution free of $\mathrm{Ca}^{2+}$ and $\mathrm{Mg}^{2+}\left(37^{\circ} \mathrm{C}\right)$ (Tanaka et al.,

Abbreviations: CPE, Cytopathic effect(s); FITC, fluorescein isothiocyanate. 
1978) containing 0.5 mM-EGTA (Sigma), 10 mM-HEPES (Nakarai Chemicals, Kyoto) and $50 \mu \mathrm{g}$ kanamycin $\mathrm{ml}^{-1}$ (Meiji Seika, Tokyo), for 8 to $10 \mathrm{~min}$ at a flow-rate of $5 \mathrm{ml} \mathrm{min}^{-1}$. Another perfusion was made for more than $20 \mathrm{~min}$ with the same solution without EGTA, containing $0.5 \mathrm{~mm}-\mathrm{CaCl}_{2}, 0.025 \%(\mathrm{w} / \mathrm{v})$ collagenase (Type IV; Sigma) and $0.005 \%(w / v)$ trypsin inhibitor (Type II-S; Sigma). Perfused liver was sliced, and a crude cell suspension was made by gentle shaking and pipetting in culture medium (described below) held at $37^{\circ} \mathrm{C}$. After filtration through cotton gauze and then stainless mesh (no. 200), the suspension was chilled on ice and washed in the medium three times by centrifuging for $1.5 \mathrm{~min}$ at $30 \mathrm{~g}$. The concentration of viable cells was adjusted to $5 \times$ $10^{5} \mathrm{ml}^{-1}$ by trypan blue exclusion and $1.5 \mathrm{ml}$ of the suspension was seeded into $35 \mathrm{~mm}$ dishes with or without glass coverslips, which were incubated at $37{ }^{\circ} \mathrm{C}$ in a humid atmosphere of $5 \% \mathrm{CO}_{2}$ in air. After 2 to $4 \mathrm{~h}$, medium containing detached cells was aspirated and replaced with fresh culture medium.

The culture medium consisted of Williams' medium E (Flow Laboratories, Rockville, Md., U.S.A.) supplemented with $10 \%$ ( $/ \mathrm{v}$ ) calf serum (Flow Laboratories), $4 \mathrm{mU}$ insulin $\mathrm{ml}^{-1}$ (Sigma), $1 \mu \mathrm{M}$-dexamethasone (Sigma) and $50 \mu \mathrm{g}$ kanamycin $\mathrm{ml}^{-1}$.

Organism. Rat-derived RT strain of Tyzzer's organism (Fujiwara et al., 1971) was maintained in mice for more than 100 passages. Infected livers with multiple focal necrosis were obtained from mice which had been inoculated intravenously with infected liver homogenate after treatment with cortisone (Fujiwara et al., 1964). Small pieces of liver were cut and stored at $-80^{\circ} \mathrm{C}$. Just before inoculation, the livers were thawed and homogenized with the kanamycin-free medium. The number of organisms in the homogenate was determined by direct microscopic counting as described previously (Fujiwara, 1967), but modified by mixing the homogenate with an equal volume of phosphate-buffered saline containing $2 \%(\mathrm{v} / \mathrm{v})$ formalin to minimize lysis of the organisms (Fujiwara, 1967).

Inoculation. After incubation of hepatocyte cultures for 20 to $30 \mathrm{~h}$, when a monolayer was formed, medium was aspirated and each dish was inoculated with $0.5 \mathrm{ml}$ of infective mouse liver homogenate containing $3 \times 10^{6}$ organisms in $1 \mathrm{ml}$ kanamycin-free medium. After incubation at $37^{\circ} \mathrm{C}$ for 2 or $24 \mathrm{~h}$, culture medium was changed to $1.5 \mathrm{ml}$ fresh kanamycin-free medium.

Observations. For macroscopic and microscopic observation, monolayers on culture dishes or on coverslips were fixed with methanol and stained with Giemsa or thionin.

The indirect immunofluorescence technique using FITC-labelled rabbit anti-mouse IgG has been described (Fujiwara et al., 1969). To prepare antiserum against RT strain, mice received intranasal inoculation of freshly infected liver homogenate and, one month later, two subcutaneous injections $4 \mathrm{~d}$ apart with formalin-fixed homogenate. Animals were bled one week after the last injection.

For electron microscopy, coverslips, showing clear cytopathic effects (CPE) were fixed in situ with $2 \cdot 5 \%$ (v/v) glutaraldehyde and then post-fixed with $1 \%(\mathrm{w} / \mathrm{v})$ osmic acid. After embedding in Spurr resin (Taab Laboratories

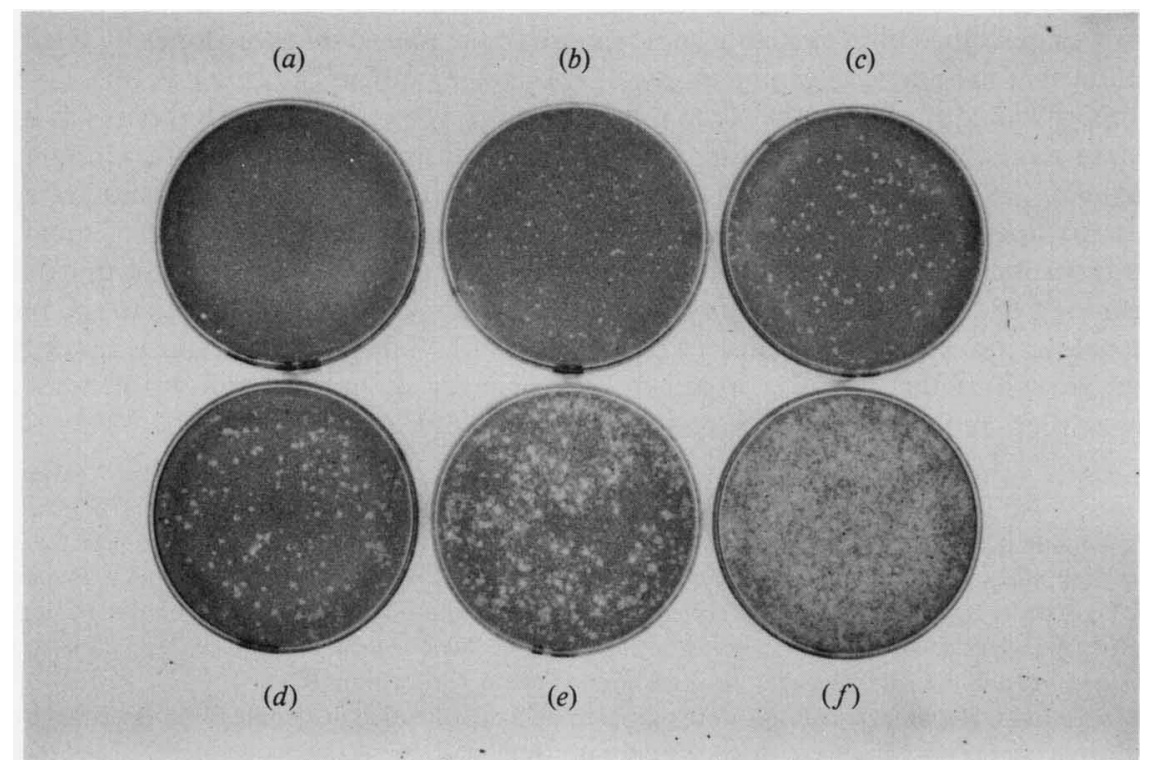

Fig. 1. Cytopathic effects on mouse hepatocyte monolayers inoculated with RT strain of Tyzzer's organism. (a) $36 \mathrm{~h},(b) 42 \mathrm{~h},(c) 48 \mathrm{~h},(d) 54 \mathrm{~h},(e) 60 \mathrm{~h}$ and $(f) 72 \mathrm{~h}$ post-infection. The monolayers were Giemsa-stained. 

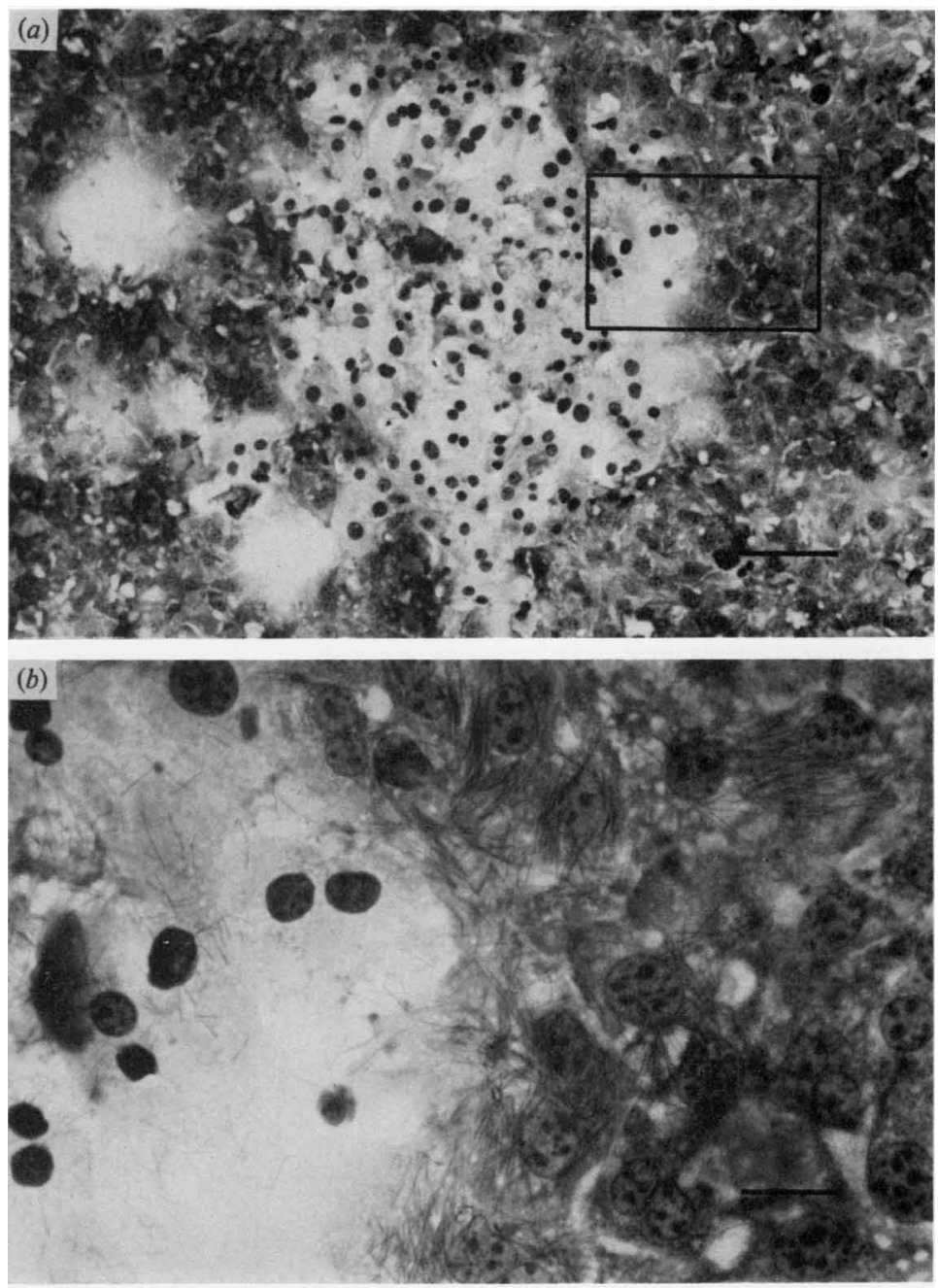

Fig. 2. Microscopy of Giemsa-stained plaque at $48 \mathrm{~h}$ post-infection. (a) Degenerated hepatocytes. The bar marker represents $100 \mu \mathrm{m}$. (b) Abundant growth of organisms within living hepatocytes and some extracellular organisms at the margin of the same plaque shown in (a) (boxed). The bar marker represents $25 \mu \mathrm{m}$.

Equipment, Reading, U.K.) and removing the coverslip, horizontal ultrathin sections of the monolayer culture were made and stained with uranium acetate and lead acetate. Specimens were examined using a JEM 100B electron microscope at $80 \mathrm{kV}$.

Growth kinetics. The number of organisms in cultures were estimated by counting of fixed, stained homogenates of whole culture or culture supernatant preparation.

Pathogenicity tests. After subcutaneous injection with $5 \mathrm{mg}$ cortisone acetate (Cortone; Merck-Banyu, Tokyo), mice were challenged intravenously with $0.2 \mathrm{ml}$ of homogenates of inoculated or uninoculated cultures. They were checked for mortality and liver lesions for $5 \mathrm{~d}$ post-inoculation.

\section{RESULTS}

\section{Observations}

Giemsa or thionin-stained preparations of inoculated hepatocyte cultures were shown to have weakly-stained spot-like plaques that could be observed macroscopically at $36 \mathrm{~h}$ post-infection (Fig. $1 a$ ). The plaques increased in size and number with incubation (Fig. $1 b, c$ ) fusing with each 

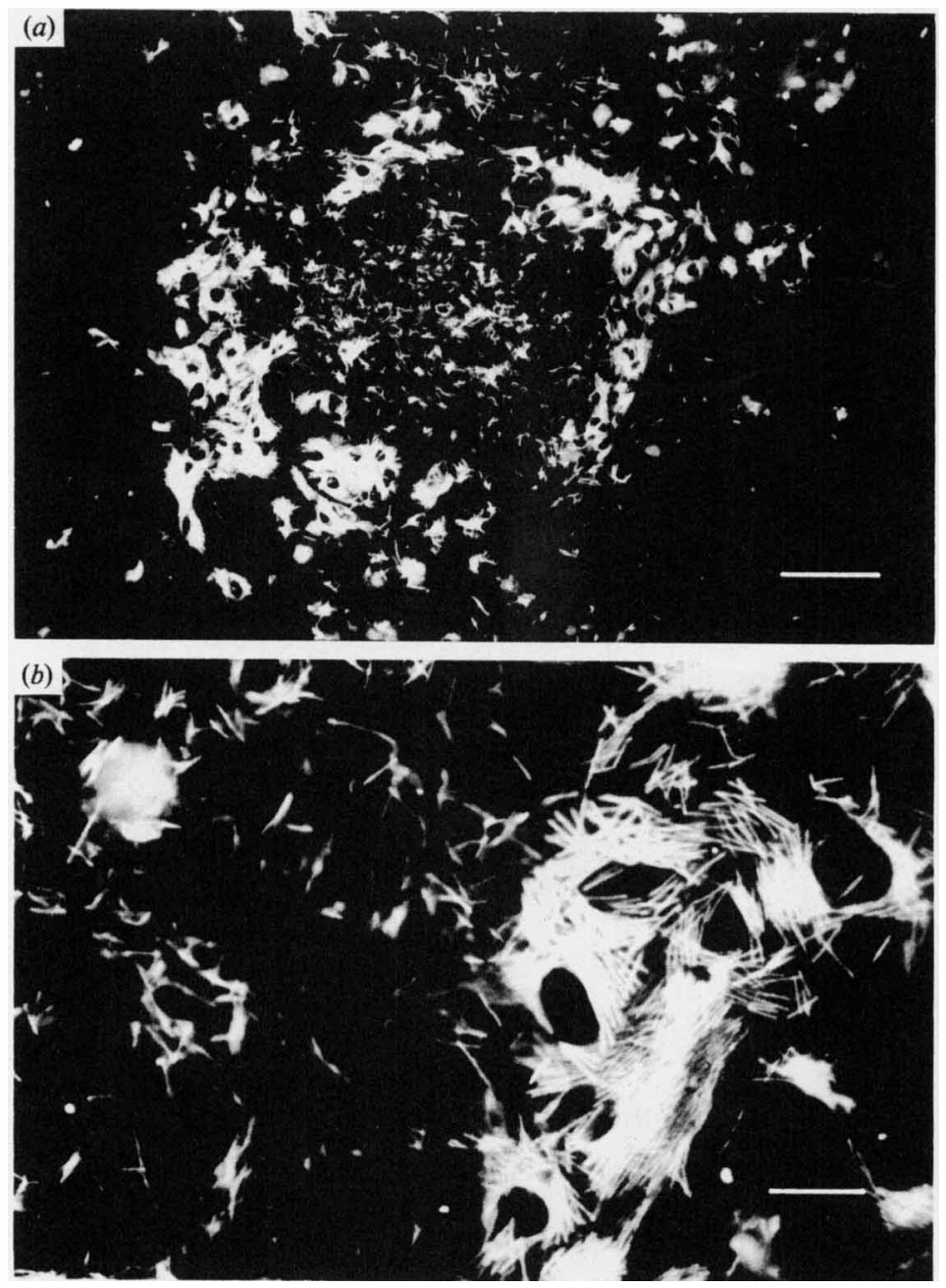

Fig. 3. FITC-positive organisms within and at the margin of a plaque. Indirect immunofluorescence with anti-RT antibody. CPE are shown $48 \mathrm{~h}$ post-infection. The bar marker represents $100 \mu \mathrm{m}$ in $(a)$ and $25 \mu \mathrm{m}$ in $(b)$.

other at 50 to $60 \mathrm{~h}$ post-infection (Fig. $1 d, e$ ). Almost the whole surface of the culture was cleared around $72 \mathrm{~h}$ post-infection (Fig. $1 f$ ).

At $48 \mathrm{~h}$ post-infection, the CPE was seen to consist of degenerated cells with pyknotic nuclei (Fig. 2a). Within the cytoplasm of apparently viable hepatocytes surrounding this region, slender bacilli were present in bundles (Fig. $2 b$ ) as seen in infected mouse liver. Some apparently extracellular organisms were also recognized among degenerated cells. On prolonged incubation, even those cells not affected by CPE were seen to be parasitized. Few cells remained uninfected at $72 \mathrm{~h}$ post-infection.

The organisms in hepatocyte cultures were stained by indirect immunofluorescence using antibody to the RT organism. The CPE at $48 \mathrm{~h}$ post-infection consisted of an area of central necrosis with a number of scattered extracellular organisms that stained with specific fluorescence, and a surrounding layer of hepatocytes which fluoresced strongly (Fig. $3 a$ ). The organisms appeared to be located intracellularly and were arranged in parallel bundles occupying the cytoplasm, but not the nuclear region of hepatocytes (Fig. $3 b$ ). Electron microscopy revealed that the organisms with flagella resided within the hepatocyte cytoplasm and that some organisms were dividing within hepatocytes (Fig. 4). 


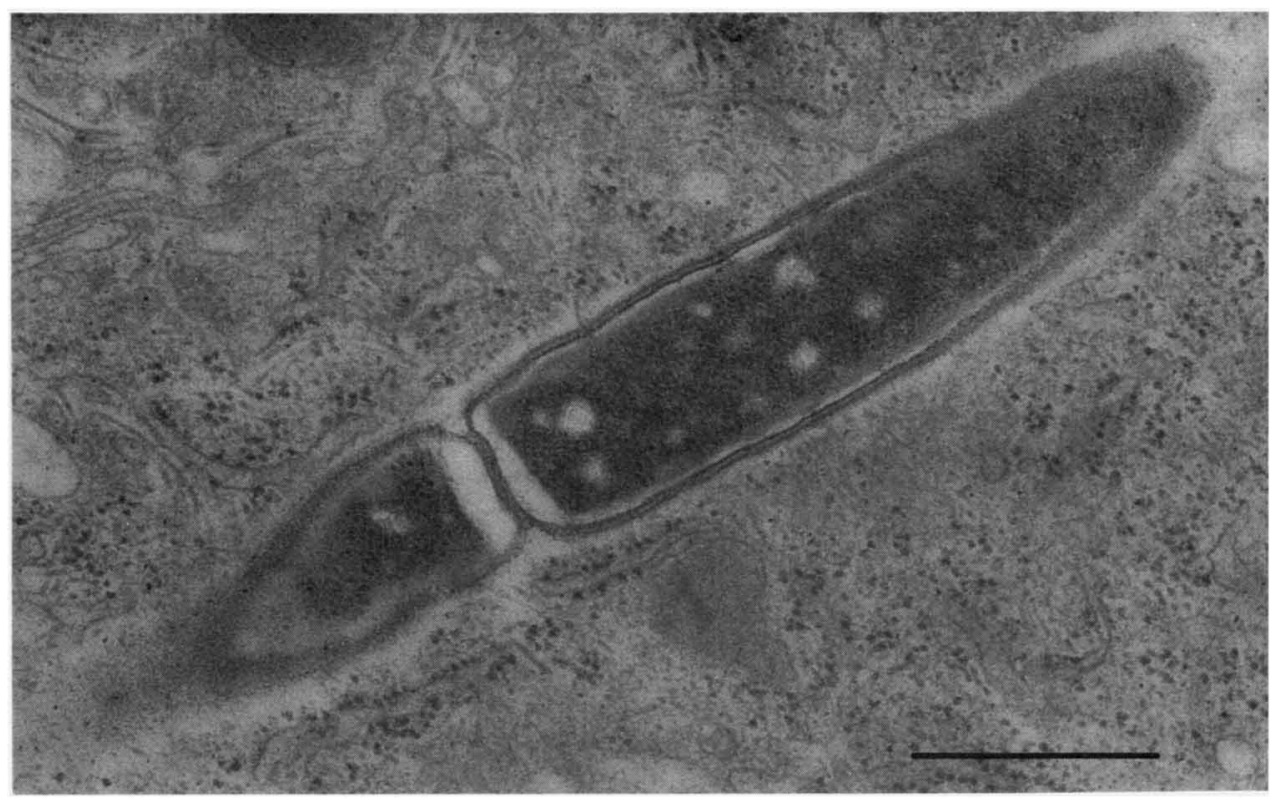

Fig. 4. Electron micrograph of an organism undergoing cell division at $54 \mathrm{~h}$ post-infection. The bar marker represents $500 \mathrm{~nm}$.

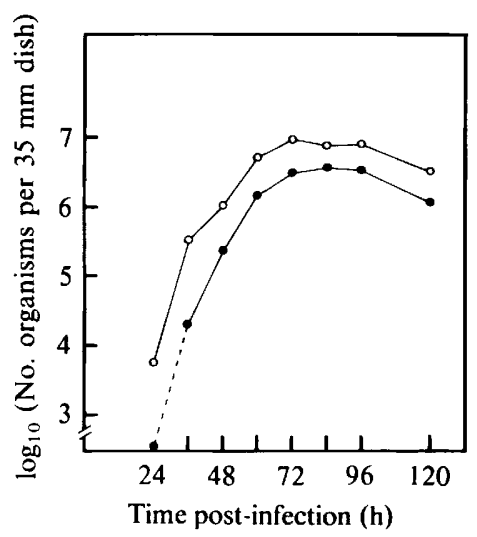

Fig. 5. Growth of RT strain of Tyzzer's organism in cultured mouse hepatocytes. Organisms $\left(3 \times 10^{6}\right.$ in $1.5 \mathrm{ml}$ medium) were added to $7.5 \times 10^{5}$ hepatocytes per $35 \mathrm{~mm}$ dish and the medium was changed once at $24 \mathrm{~h}$ post-infection. $\mathrm{O}$, Counts in whole culture; $O$, counts in culture supernatant.

\section{Growth kinetics}

A growth curve of the organisms in cultured hepatocytes was obtained by microscopic counting (Fig. 5). At $24 \mathrm{~h}$ post-infection, that is, just after the inoculum medium was changed, the number of organisms in the whole culture was only $10^{3}$ organisms $\mathrm{ml}^{-1}$. Later, organisms rapidly increased in number reaching a maximum around $72 \mathrm{~h}$ post-infection, and gradually declined thereafter. The number of organisms in culture supernatants was one third of that in the whole culture throughout the observation period.

\section{Pathogenicity tests}

The results obtained from the two experiments (I and II) are summarized in Table 1. While homogenates of the whole cultures sampled at $12 \mathrm{~h}$ post-infection or earlier had no effect, those sampled at 24 to $48 \mathrm{~h}$ post-infection were lethal for mice. The lethal effect decreased after $96 \mathrm{~h}$ 
Table 1. Pathogenicity for mice of RT strain of Tyzzer's organism grown in mouse hepatocyte culture

Female ICR mice (4-5 weeks old) were challenged intravenously with $0.2 \mathrm{ml}$ of homogenate of infected hepatocytes prepared by adding $3 \times 10^{6}$ organisms in $1.5 \mathrm{ml}$ medium to $7.5 \times 10^{5}$ hepatocytes per 35 $\mathrm{mm}$ dish; medium was changed once $2 \mathrm{~h}$ (experiment $\mathrm{I}$ ) or $24 \mathrm{~h}$ (experiment II) post-infection. Control animals received homogenate of uninfected hepatocytes.

\begin{tabular}{|c|c|c|c|c|c|c|}
\hline \multirow{3}{*}{$\begin{array}{l}\text { Time after } \\
\text { infection of } \\
\text { hepatocytes } \\
\text { (h) }\end{array}$} & \multicolumn{3}{|c|}{ Experiment I } & \multicolumn{3}{|c|}{ Experiment II } \\
\hline & \multirow{2}{*}{$\begin{array}{c}\text { No. of organisms } \\
\text { (ml culture } \\
\text { homogenate) })^{-1}\end{array}$} & \multicolumn{2}{|c|}{$\begin{array}{l}\text { Mortality* of mice } \\
\text { receiving: }\end{array}$} & \multirow{2}{*}{ 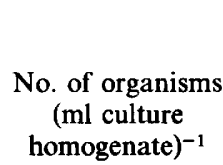 } & \multicolumn{2}{|c|}{$\begin{array}{l}\text { Mortality* of mice } \\
\text { receiving: }\end{array}$} \\
\hline & & $\begin{array}{l}\text { Infected } \\
\text { culture }\end{array}$ & $\begin{array}{l}\text { Control } \\
\text { culture }\end{array}$ & & $\begin{array}{l}\text { Infected } \\
\text { culture }\end{array}$ & $\begin{array}{l}\text { Control } \\
\text { culture }\end{array}$ \\
\hline 2 & $4 \times 10^{4}$ & $0 / 5$ & & & & \\
\hline 6 & $4 \times 10^{4}$ & $0 / 5$ & & & & \\
\hline 12 & $8 \times 10^{3}$ & $0 / 5$ & $0 / 5$ & & & \\
\hline 24 & $4 \times 10^{3}$ & $5 / 5$ & & $2.0 \times 10^{4}$ & $5 / 5$ & $0 / 5$ \\
\hline 36 & $1.0 \times 10^{5}$ & $5 / 5$ & & $9.2 \times 10^{4}$ & $5 / 5$ & \\
\hline 48 & $8.4 \times 10^{5}$ & $5 / 5$ & $0 / 5$ & $1.5 \times 10^{6}$ & $5 / 5$ & $0 / 5$ \\
\hline 72 & & & & $5.4 \times 10^{6}$ & $5 / 5$ & $0 / 5$ \\
\hline 84 & & & & $6.2 \times 10^{6}$ & $5 / 5$ & \\
\hline 96 & & & & $4.8 \times 10^{6}$ & $2 / 5$ & \\
\hline 120 & & & & $2.7 \times 10^{6}$ & $0 / 5$ & $0 / 5$ \\
\hline 144 & & & & $8.4 \times 10^{5}$ & $0 / 5$ & \\
\hline
\end{tabular}

* Mortality is defined as (no. of dead mice)/(no. of tested mice) at $5 \mathrm{~d}$ post-infection.

post-infection; this correlated with a decline in the numbers of visible organisms. Affected mice showed multiple focal necrosis of the liver with the presence of organisms within hepatocytes. Organisms from the liver of affected mice could be cultured in hepatocytes. No liver lesions were produced in mice injected with uninoculated hepatocyte cultures.

\section{DISCUSSION}

The successful propagation of the Tyzzer's organism in tissue culture was described by Rights et al. (1947), who achieved abundant growth of the organisms in mouse embryo tissue placed on agar slopes. With chick embryo tissue, however, the results were not satisfactory. Craigie (1966) failed to infect a monolayer culture of the yolk sac endoderm from chick embryos, although some parasitized cells were seen when the culture was prepared from infected embryos. He attributed his failure to unsuccessful penetration of the organism into cultured cells rather than alteration in cell metabolism in culture.

In the present study, attempts were made to grow the Tyzzer's organism in a monolayer culture in order to obtain a convenient system for in vitro study. The criteria for in vitro propagation of the organism during the present studies were: (1) morphological changes in inoculated hepatocyte cultures, (2) presence of organisms in hepatocytes, (3) positive reaction of the organisms by immunofluorescence, (4) a progressive increase in bacterial concentration in infected hepatocytes and (5) production of the disease in mice inoculated with homogenates prepared from infected hepatocyte cultures. Since these criteria were fulfilled, our conclusion was that the Tyzzer's organisms actually grew in a monolayer culture of adult mouse hepatocytes. Since Tyzzer (1917), the organism has been known to be a strict intrahepatocytic parasite and the present study revealed that it appeared to grow within cytoplasm of cultured hepatocytes.

Both macroscopically and microscopically, CPE produced in the infected monolayer cultures showed characteristics similar to liver lesions seen in vivo. The organisms formed plaque-like CPE in monolayer cultures without agar overlay and the affected area was surrounded by infected hepatocytes. These findings strongly support the idea that cell-to-cell spread occurs in vivo. However, as the extent of CPE increased with prolonged incubation, it is probable that 
organisms released extracellularly from damaged hepatocytes also infect other intact cells in culture. The present in vitro system might provide a great deal of information on cytopathogenicity of the organism.

The growth curve of organisms obtained in this study showed direct evidence of multiplication of the organisms in the culture. In view of the extreme fragility of these organisms in infected mouse liver homogenate (Fujiwara, 1967; Fujiwara et al., 1973), the presence of $10^{6}$ or more organisms $\mathrm{ml}^{-1}$ in the culture supernatant at $60 \mathrm{~h}$ post-infection or later can be considered as resulting from abundant growth of the organisms. In addition, the virulence of the propagated organisms for mice was demonstrated as early as $24 \mathrm{~h}$ post-infection as well as at $84 \mathrm{~h}$ post-infection. Such yield of infective organisms is of great advantage for preparing infective materials or specific antigens under controllable conditions compared to materials from infected mice.

Quantitative estimation of the organisms in experimental studies has been based upon direct microscopic counting of stained preparations (Fujiwara, 1967). By this method, however, it is impossible to determine the number of viable or infective organisms. Attempts were made to evaluate infectivity according to mean survival time of infected chick embryos (Craigie, 1966), $50 \%$ lethal dose for embryonated eggs (Ganaway et al., 1971 a), or mortality of inoculated mice (Fujiwara et al., 1973). The present finding of CPE in inoculated monolayer cultures may provide another method of quantitative estimation by plaque assay.

\section{REFERENCES}

ARnheiter, H. (1980). Primary monolayer culture of adult mouse hepatocytes - a model for the study of hepatotropic viruses. Archives of Virology 63, 11-22.

Craigie, J. (1966). 'Bacillus piliformis' (Tyzzer) and Tyzzer's disease of the laboratory mouse. I. Propagation of the organism in embryonated eggs. Proceedings of the Royal Society B165, 35-60.

FujIWARA, K. (1967). Complement fixation reaction and agar gel double diffusion test in Tyzzer's disease of mice. Japanese Journal of Microbiology 11, 103117.

Fujiwara, K. (1971). Problems in checking inapparent infections in laboratory mouse colonies: an attempt at serological checking by anamnestic response. In Defining of Laboratory Animals, pp. 7792. Edited by H. A. Schneider. Washington, D.C.: National Academy of Sciences.

FujIWARA, K. (1978). Tyzzer's disease. Japanese Journal of Experimental Medicine 48, 467-480.

Fujiwara, K., Takagaki, Y., Maejima, K., Kato, K., NAIKI, M. \& TAJIMA, Y. (1963). Tyzzer's disease in mice: pathologic studies on experimentally infected animals. Japanese Journal of Experimental Medicine 33, 183-202.

Fujiwara, K., Takagaki, Y., NaIki, M., Maejima, K. \& Tajima, Y. (1964). Tyzzer's disease in mice. Effects of corticosteroids on the formation of liver lesions and the level of blood transaminases in experimentally infected animals. Japanese Journal of Experimental Medicine 34, 59-75.

Fujiwara, K., Takahashi, R., Kurashina, H. \& Matsunuma, N. (1969). Protective serum antibodies in Tyzzer's disease of mice. Japanese Journal of Experimental Medicine 39, 491-504.
Fujiwara, K., Yamada, A., Ogawa, H. \& Oshima, Y. (1971). Comparative studies on the Tyzzer's organisms from rats and mice. Japanese Journal of Experimental Medicine 41, 125-133.

Fujiwara, K., Kurashina, H., Magaribuchi, T., Takenaka, S. \& Yokoryama, S. (1973). Further observations on the difference between Tyzzer's organisms from mice and those from rats. Japanese Journal of Experimental Medicine 43, 307-315.

Ganaway, J. R., Allen, A. M. \& Moore, T. D. $(1971 a)$. Tyzzer's disease of rabbits: isolation and propagation of Bacillus piliformis (Tyzzer) in embryonated eggs. Infection and Immunity 3, 429-437.

Ganaway, J. R., Allen, A. M. \& Moore, T. D. $(1971 b)$. Tyzzer's disease. American Journal of Pathology 64, 717-732.

Kanazawa, K. \& Imai, A. (1959). Pure culture of the pathogenic agent of Tyzzer's disease of mice. Nature, London 184, 1810-1811.

Rights, F. L., JACKson, E. B. \& Smadel, J. E. (1947). Observations on Tyzzer's disease in mice. American Journal of Pathology 23, 627-635.

SEgLEN, P. O. (1976). Preparation of isolated rat liver cells. Methods in Cell Biology 13, 29-83.

TANAKa, K., Sato, M., Tomita, Y. \& Ichihara, A. (1978). Biochemical studies on liver functions in primary cultured hepatocytes of adult rats. I. Hormonal effects on cell viability and protein synthesis. Journal of Biochemistry 84, 937-946.

Tyzzer, E. E. (1917). A fatal disease of the Japanese waltzing mouse caused by a spore-bearing bacillus (Bacillus piliformis N. sp.). Journal of Medical Research 37, 307-338. 